\title{
A Method for Culturing Mouse Whisker Follicles to Study Circadian Rhythms ex vivo
}

Atsuhiro Nishida ${ }^{1}$, Yoshiki Miyawaki ${ }^{1}, K^{\prime}$ oichi Node ${ }^{2}$ and Makoto Akashi ${ }^{1}$ *

${ }^{1}$ The Research Institute for Time Studies, Yamaguchi University, 1677-1 Yoshida, Yamaguchi, Yamaguchi, Japan; ²Department of Cardiovascular Medicine, Saga University, 5-1-1 Nabeshima, Saga, Japan

*For correspondence: akashima@yamaguchi-u.ac.jp

\begin{abstract}
[Abstract] Ex vivo tissue-culture experiments are often performed in the field of circadian biology. The major aim of these experiments is to evaluate circadian characteristics such as period length at the tissue-autonomous level by monitoring clock gene expression in real time. This culture method is also used to examine the tissue specificity of circadian entrainment factors. However, an ex vivo culture method for monitoring clock gene expression in hair follicles has yet to be established. In the present study, we developed an experimental method to analogize and evaluate circadian characteristics by performing ex vivo culture of mouse whisker follicles and monitoring clock gene expression in real time.
\end{abstract} Keywords: Circadian rhythm, Clock gene, Hair follicle, Ex vivo culture, Luciferase, Mouse

[Background] Almost all living organisms exhibit physiological and behavioral circadian rhythms that are driven by the circadian clock (Takahashi, 2017). The circadian clock enables maximum expression of genes at appropriate times of the day, allowing organisms to appropriately adapt to environmental rhythms generated by the Earth's rotation. The clockwork consists of ubiquitous, cell-autonomous and clock gene-driven negative feedback loops of transcription (Schibler et al., 2015). In mammals, the transcription factors BMAL1 and CLOCK activate the transcription of clock and clock-related genes such as Period (Per) and Cryptochrome (Cry) via E-box elements. PER, together with CRY, a potent transcriptional inhibitor, subsequently function to negatively regulate this complex (Kume et al., 1999).

Ex vivo tissue-culture experiments are often performed in the field of circadian biology (Yamazaki et al., 2000). The major aim of these experiments is to evaluate the circadian characteristics of clock gene expression such as period length at the tissue-autonomous level and to compare these characteristics with those at the whole-body level (Liu et al., 2007). For example, the effect of the dysfunction of a clock gene in question can be investigated by comparing the effects in ex vivo tissue culture and behavior and physiology. This culture method is also used to examine the tissue specificity of circadian entrainment factors (Sato et al., 2014). Specifically, this technique can be used to reveal in which tissue a humoral factor in question modulates circadian phase or amplitude. Additionally, although controversial, some studies suggest that the circadian phase observed in ex vivo cultured tissues can be used to estimate that in vivo (Stokkan et al., 2001).

In the present study, we developed an experimental method to analogize and evaluate circadian characteristics based on ex vivo culture of mouse whisker follicles. Briefly, individual whisker follicles are carefully dissected from mice carrying a luciferase gene whose expression is driven by a circadian 
promoter, and bioluminescence is measured in real time using a photomultiplier tube. This method can be useful for a wide range of applications as mentioned above.

\section{Materials and Reagents}

1. $100 \mathrm{~mm}$ Petri dish (IWAKI, catalog number: SH90-20)

2. $35 \mathrm{~mm}$ culture dish (IWAKI, catalog number: 1000-035)

3. Eppendorf tube

4. Inbred mice such as male 5-20-week-old C57/BL6 mice carrying the luciferase gene driven by a clock gene promoter (in our original paper, we used Per2::luc knock-in mice and Bmal1-Eluc transgenic mice, which were gifts from Dr. Joseph Takahashi and Dr. Yoshihiro Nakajima, respectively) (Yoo et al., 2004; Noguchi et al., 2012a)

5. Silicone (Shin-Etsu, catalog number: KS-64)

6. $70 \%$ ethanol (Shinwa Alcohol Industry, catalog number: 4079210060 )

7. Phosphate-buffered saline (PBS) (Nacalai Tesque, catalog number: 14249-24)

8. DMEM (Nacalai, catalog number: 08456-94)

9. Penicillin/streptomycin (Thermo Fisher Scientific, Gibco ${ }^{\mathrm{TM}}$, catalog number: 15070-063)

10. Dexamethasone (Sigma-Aldrich, catalog number: D4902)

11. Luciferin (WAKO, catalog number: 126-05116)

12. Dimethyl sulfoxide

13. DMEM supplemented with penicillin/streptomycin (see Recipes)

14. Luciferin-containing medium (see Recipes)

15. $1,000 x$ DEX stock (see Recipes)

\section{Equipment}

1. Surgical scissors (Hammacher, catalog number: 91-1538)

2. Claw tweezers (FST, catalog number: 11154-10)

3. Fine tweezers (FST, catalog number: 18132-12)

4. Laminar flow cabinet (SANYO, model: MCV-B131F)

5. $\mathrm{CO}_{2}$ incubator with an infrared sensor that is not affected by humidity inside the chamber (ASTEC, model: SCA-165DRS)

6. Photomultiplier tube (Hamamatsu, model: LM2400)

7. Dissecting microscope (Nikon, model: SMZ745T)

\section{Software}

1. Software for Photon Detection Unit C10749(LM2400v21)-JP

2. Cosinor software 


\section{Procedure}

\section{Notes:}

1. Although the absolute value of circadian period length in cultured hair follicles differs from those obtained from physiological and behavioral studies, we confirmed that the relative difference in period length among mouse genotypes is similar between clock gene expression in hair follicles and locomotor activity (Yamaguchi et al., 2017). Our ex vivo methods may, therefore, be useful tools for analogizing in vivo circadian characteristics.

2. Because culture medium composition reportedly affects period length (Lee et al., 2011; Noguchi et al., 2012b), it is important to use identical medium composition and product lot numbers throughout all sets of experiments for comparison of relative differences between animals. For example, we have found that the absence of phenol red results in damping and relatively longer periods.

3. Dexamethasone (DEX) is a well-known synchronizer for peripheral clocks. Therefore, clock gene expression is often monitored after DEX treatment.

A. Isolation of mouse whisker follicles

1. All protocols for animal experiments must be approved by an institutional animal research committee. Animal studies must be performed in compliance with institutional animal care and use guidelines. Figure 1 indicates a clean environment and tools required for following experimental procedures.

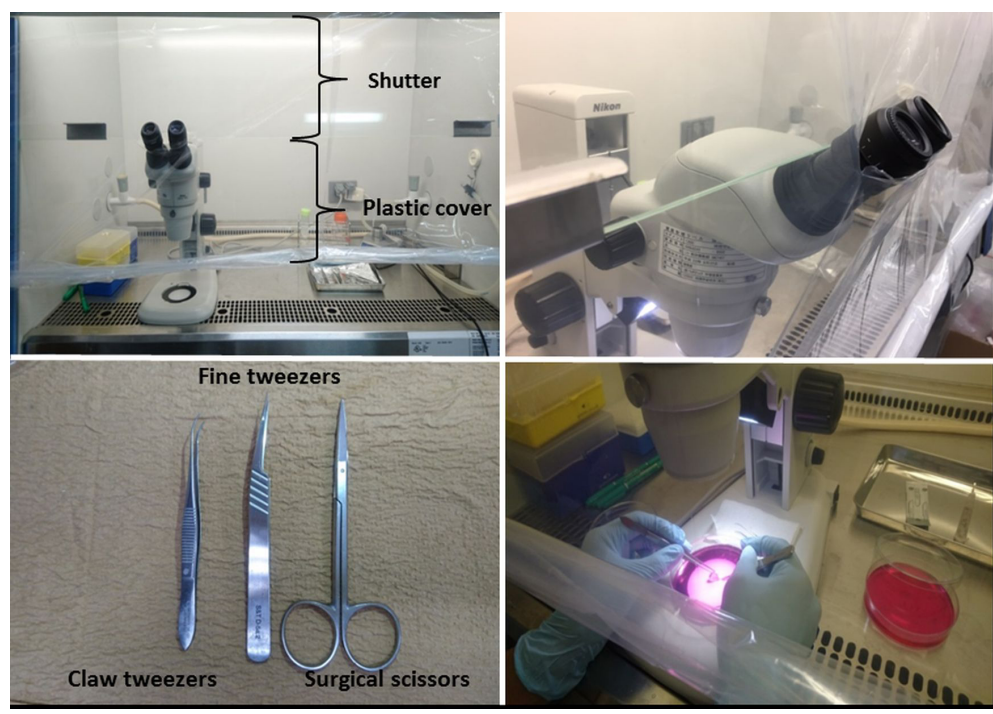

Figure 1. Example of a clean environment and tools required for the experimental procedures

2. Maintain mice carrying the luciferase gene driven by circadian promoter/enhancer elements on a 12-h light-dark (LD) cycle and allow ad libitum access to food and water. 
3. After euthanasia, vigorously wipe both the left and right mystacial pads with $70 \%$ ethanol and remove them from the mice with surgical scissors by making incisions in the recommended order indicated in Figure 2. To avoid damaging hair follicles, insert surgical scissors and cut along the interface between the skin and bone tissue.

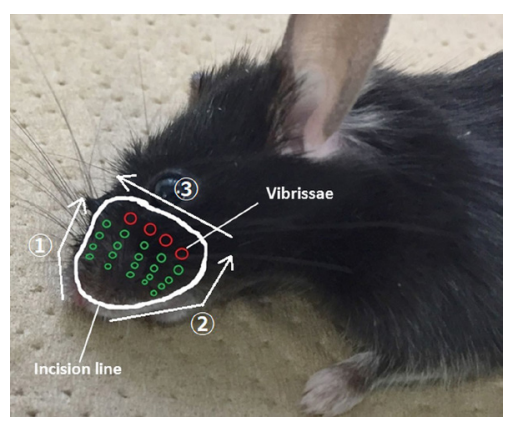

Figure 2. The mystacial pad on a mouse and the incision line

4. On a clean bench, vigorously wash the pads two times with $70 \%$ ethanol and three times with phosphate-buffered saline (PBS) for about $15 \mathrm{~s}$ each (Video 1). Minimize carry-over contamination in each step.

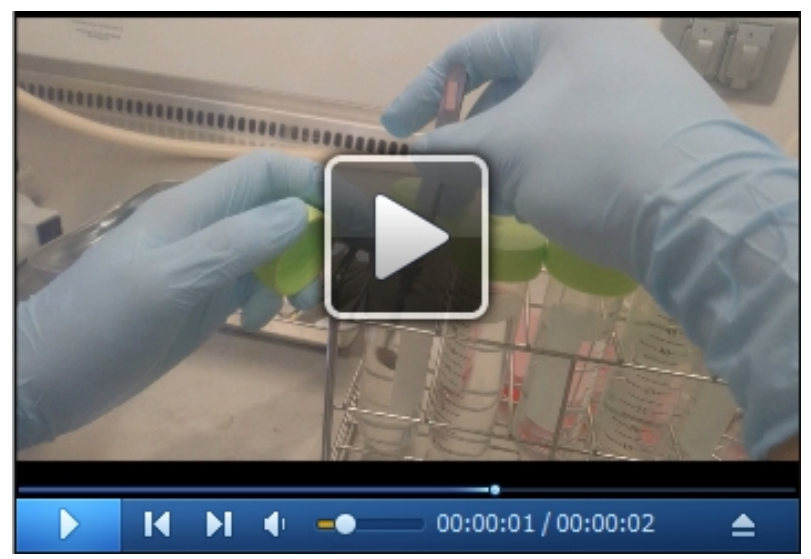

Video 1. Vigorous washing of mystacial pads

5. Transfer the pads to a 100-mm Petri dish containing $25 \mathrm{ml}$ fresh DMEM supplemented with penicillin and streptomycin at room temperature.

6. On a clean bench, carefully dissect individual whisker follicles as described below under a dissecting microscope (Recommended magnification: 10x).

7. Use claw tweezers to carefully remove the surrounding connective tissue without damaging the hair follicles (Figure $3 \mathrm{~A}$ and Video 2). Denude the hair follicles as thoroughly as possible (Figure 3B). 

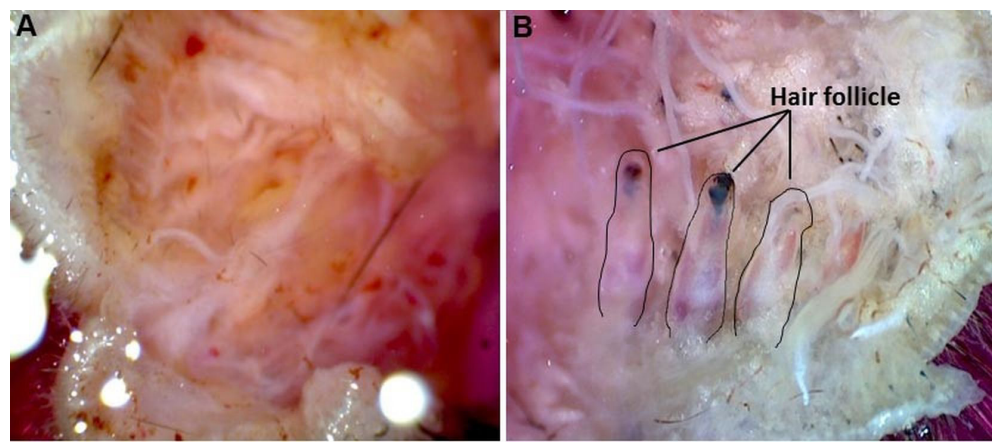

Figure 3. Dissection of individual whisker follicles. A. Image of the reverse side of a removed mystacial pad. Hair follicles are invisible because they are covered by connective tissue. Use claw tweezers to carefully remove the connective tissue of the mystacial pad without damaging the hair follicles. B. Black outlines indicate denuded hair follicles after removal of the connective tissue. Denude the hair follicles as thoroughly as possible.

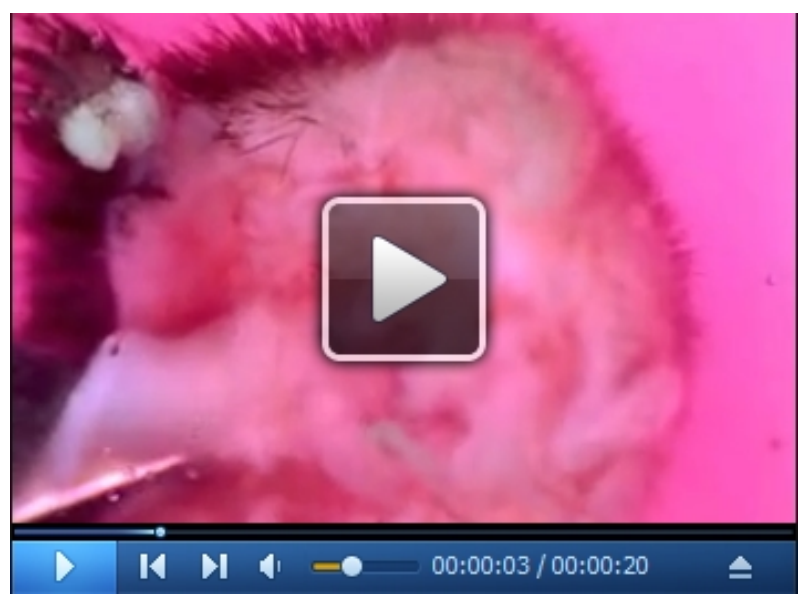

Video 2. Removing connective tissue of the mystacial pad (This video was made at Yamaguchi Univ. according to guidelines from the Yamaguchi Univ. on Animal Care and approved by the Animal Research Ethics Board of Yamaguchi University under protocol \#298.)

8. Hold the root of a hair follicle firmly with fine tweezers and pluck it out from the skin, being careful not to damage the hair follicle (Video 3). 


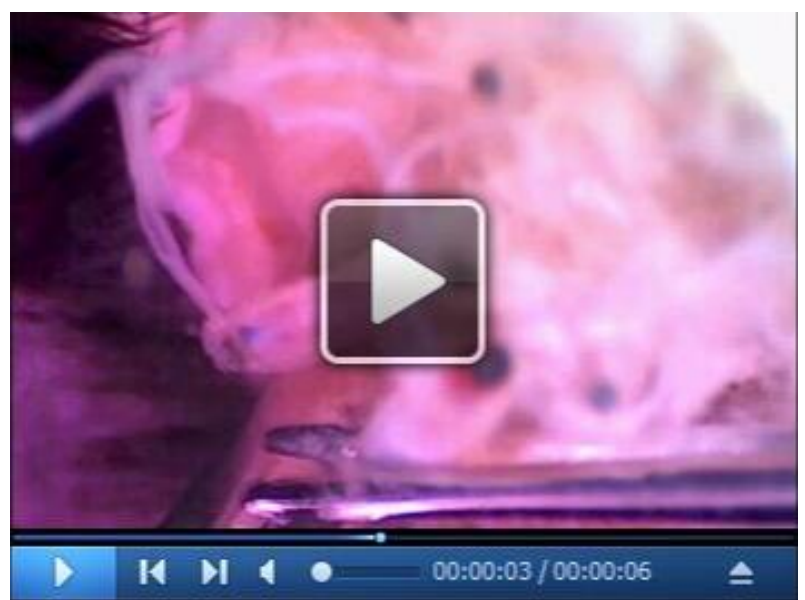

Video 3. Plucking a hair follicle from the skin (This video was made at Yamaguchi Univ. according to guidelines from the Yamaguchi Univ. on Animal Care and approved by the Animal Research Ethics Board of Yamaguchi University under protocol \#298.)

B. Ex vivo culture of mouse whisker follicles to examine circadian characteristics

1. Transfer isolated whisker follicles to another $100-\mathrm{mm}$ Petri dish containing $25 \mathrm{ml}$ fresh DMEM supplemented with penicillin and streptomycin at room temperature.

2. (Optional) Classify the hair cycle stage of whisker follicles as anagen or catagen according to the morphology of the hair bulb and relative length of the hair shaft by referring to previous reports (lida et al., 2007). We previously investigated the effect of the differences in hair stage on circadian period length and found no significant differences in period length among stages.

3. Place 50-100 $\mu \mathrm{l}$ silicone into a mound somewhat off-center on the bottom of $35-\mathrm{mm}$ culture dishes. Sterilization of silicone is not essential, but we recommend using silicone from an unopened packet designated for culture use.

4. Cut the hair shaft, leaving about 10 to $20 \mathrm{~mm}$ (Figure 4A).

5. Transfer the whisker follicles to the 35-mm culture dishes for bioluminescence monitoring. Use one dish per whisker follicle. To avoid floating during bioluminescence monitoring, push the hair shaft into the silicone mound so that the shaft is stuck to the silicone and is fixed on the bottom of the dish (Figure 4B). Position the hair follicle near the center of the dish for efficient bioluminescence detection (Figure $4 \mathrm{C}$ ). 

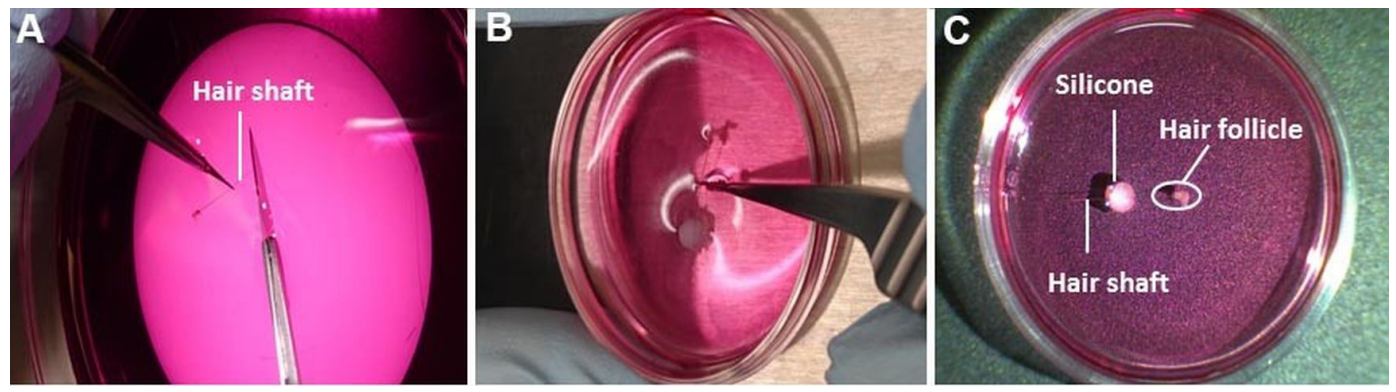

Figure 4. Fixing a hair follicle to the bottom of a culture dish. A. Cut the hair shaft, leaving about 10 to $20 \mathrm{~mm}$. B. Push the hair shaft into the silicone mound so that the shaft is stuck to the silicone and is fixed to the bottom of the dish. C. Position the hair follicle near the center of the dish.

6. Cover immobilized hair follicles with $3 \mathrm{ml}$ fresh DMEM supplemented with penicillin and streptomycin, and pre-culture at $35{ }^{\circ} \mathrm{C}$ with $5 \% \mathrm{CO}_{2}$ to allow them to recover from surgical damage. There are no specific parameters for confirming whether or not hair follicles are healthy.

7. After 1 or 2 days of pre-culture, induce circadian synchronization by adding $100 \mathrm{nM}$ dexamethasone (DEX) without replacing the culture medium and incubating for $2 \mathrm{~h}$. To facilitate mixing of DEX with culture medium, pipette $500 \mu \mathrm{l}$ of medium from each culture dish, mix this with $3 \mu \mathrm{LEX}$ (100 $\mu \mathrm{M}$ stock) by pipetting in an Eppendorf tube and add this mixture to the original culture dish.

8. During DEX treatment, prepare luciferin-containing medium at a concentration of $0.1 \mathrm{mM}$.

9. Aspirate the DEX-containing medium and wash the hair follicles with $3 \mathrm{ml}$ fresh DMEM to remove DEX, and cover the follicles with $3 \mathrm{ml}$ luciferin-containing medium. Bioluminescence is detectable for a period of more than four days if hair follicles are healthy.

10. Measure bioluminescence in real time using a photomultiplier tube inside a dark box specifically designed to reduce background noise to detect ultra-weak photon emissions (LM2400, Hamamatsu) at $35^{\circ} \mathrm{C}$ with $5 \% \mathrm{CO}_{2}$ (Figure $5 \mathrm{~A}$ ). To avoid rust formation, the LM2400 is located inside a $\mathrm{CO}_{2}$ culture incubator under low humidity conditions: to prevent culture medium from drying out, instead of using the water tray from the $\mathrm{CO}_{2}$ incubator, use the one inside the LM2400 (Figures $5 \mathrm{~B}$, sterile water). Use a $\mathrm{CO}_{2}$ incubator that utilizes an infrared sensor that is not affected by humidity inside the chamber. For measurement, open the top of the LM2400 and simply place culture dishes on the metal tray (Figures $5 B$ and $5 C$ ). Culture dishes without hair follicles should provide reads of 5,000-10,000 counts per minute (negative control). Small lung explants (1-2 $\left.\mathrm{mm}^{3}\right)$ are recommended as a positive control because samples can easily be prepared by simply cutting lung tissue with a surgical knife, and the success rate for obtaining clear circadian oscillations of bioluminescence is high ( $>80 \%$ ). The success rate for detecting clear circadian rhythmicity is dependent on a number of factors such as mouse age, sample handling, and tissue type. Therefore, the required number of sample replicates differs among experiments. 

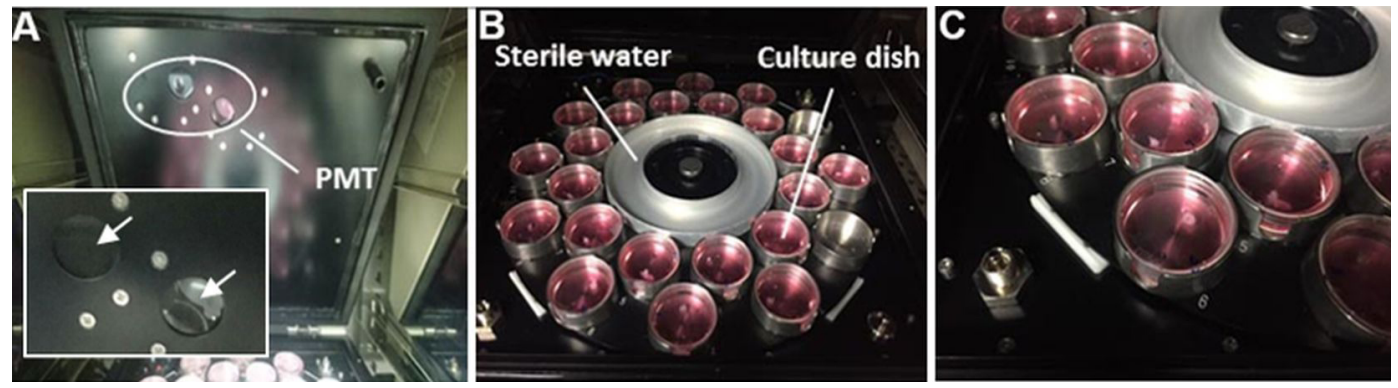

Figure 5. Monitoring bioluminescence in real time. Bioluminescence can be measured in real time using a photomultiplier tube $(\mathrm{LM} 2400)$ at $35{ }^{\circ} \mathrm{C}$ with $5 \% \mathrm{CO}_{2}$. A. The location of photomultiplier tubes (PMTs). Arrows in the inset indicate the photon input windows in a magnified view of two PMTs. B. Culture dishes on the metal tray inside the LM2400. C. Partial enlarged image of B.

\section{Data analysis}

Data collection is performed using the associated software "Software for Photon Detection Unit C10749(LM2400v21)-JP”. To analyze circadian parameters, baseline changes need to be removed. Raw data sets are therefore detrended, as shown in Figure 6, using Microsoft Excel by subtracting the 24-h running average from the raw data (Figure 6A, raw data; Figure 6B, detrended data). Circadian robustness, circadian phase (angle) and circadian period length are calculated using detrended data and Cosinor software provided by Dr. Refinetti.
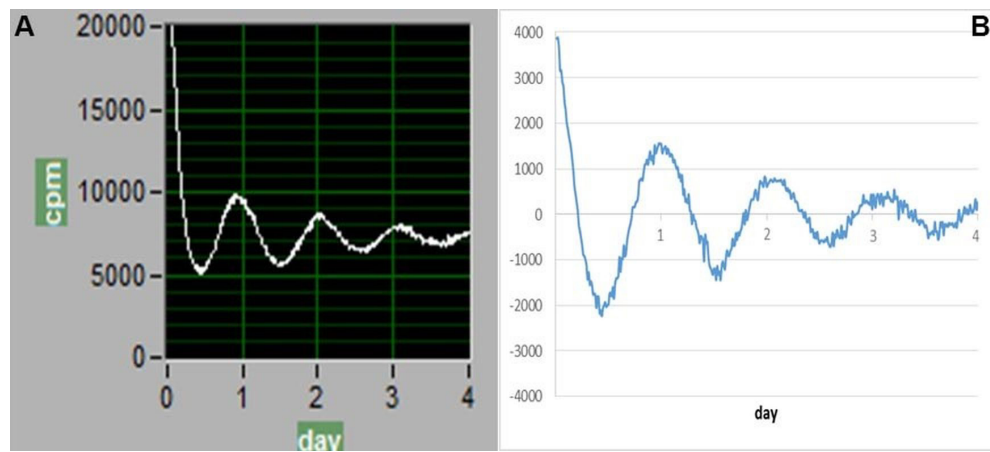

Figure 6. Example data. Data sets are detrended by subtracting the 24-h running average from the raw data. A. Raw data (vertical axis: raw counts per minute); B. Detrended data (vertical axis: detrended relative counts per minute).

\section{Recipes}

1. DMEM supplemented with penicillin and streptomycin DMEM

$1 \%$ penicillin/streptomycin 
2. Luciferin-containing medium (no filtration required)

\section{DMEM}

$1 \%$ penicillin/streptomycin

$0.1 \mathrm{mM}$ luciferin (use $10 \mathrm{mM}$ stock dissolved in saline)

3. $1,000 \times$ DEX stock (no filtration required)

Dimethyl sulfoxide

$100 \mu \mathrm{M}$ dexamethasone

\section{Acknowledgments}

We thank Ritsuko Matsumura, Rie Okamitsu and Junko Sumino for their expert technical assistance. We express our great appreciation to Takashi Matsuzaki (Shimane University) and Roberto Refinetti (Boise State University) for technical support and Cosinor software, respectively. This protocol was originally developed in Yamaguchi et al. (2017). We acknowledge the support of fellowships from the Yamaguchi Gerontology Research Institute, the Akaeda Medical Research Foundation, the SENSHIN Medical Research Foundation, and the Japan Society for the Promotion of Science.

\section{Competing interests}

The authors declare no competing financial interests.

\section{Ethics}

All protocols for animal experiments were approved by the Animal Research Committee of Yamaguchi University. Animal studies were performed in compliance with the Yamaguchi University Animal Care and Use guidelines.

\section{$\underline{\text { References }}$}

1. lida, M., Ihara, S. and Matsuzaki, T. (2007). Hair cycle-dependent changes of alkaline phosphatase activity in the mesenchyme and epithelium in mouse vibrissal follicles. Dev Growth Differ 49(3): 185-195.

2. Kume, K., Zylka, M. J., Sriram, S., Shearman, L. P., Weaver, D. R., Jin, X., Maywood, E. S., Hastings, M. H. and Reppert, S. M. (1999). mCRY1 and mCRY2 are essential components of the negative limb of the circadian clock feedback loop. Cell 98(2): 193-205.

3. Lee, S. K., Achieng, E., Maddox, C., Chen, S. C., luvone, P. M. and Fukuhara, C. (2011). Extracellular low $\mathrm{pH}$ affects circadian rhythm expression in human primary fibroblasts. Biochem Biophys Res Commun 416(3-4): 337-342. 
Please cite this article as: Nishida et. al., (2019). A Method for Culturing Mouse Whisker Follicles to Study Circadian Rhythms ex vivo,Bio-protocol 9 (2):

4. Liu, A. C., Welsh, D. K., Ko, C. H., Tran, H. G., Zhang, E. E., Priest, A. A., Buhr, E. D., Singer, O., Meeker, K., Verma, I. M., Doyle, F. J., 3rd, Takahashi, J. S. and Kay, S. A. (2007). Intercellular coupling confers robustness against mutations in the SCN circadian clock network. Cell 129(3): 605-616.

5. Noguchi, T., Ikeda, M., Ohmiya, Y. and Nakajima, Y. (2012a). A dual-color luciferase assay system reveals circadian resetting of cultured fibroblasts by co-cultured adrenal glands. PLoS One 7(5): e37093.

6. Noguchi, T., Wang, C. W., Pan, H. and Welsh, D. K. (2012b). Fibroblast circadian rhythms of PER2 expression depend on membrane potential and intracellular calcium. Chronobiol Int 29(6): 653-664.

7. Sato, M., Murakami, M., Node, K., Matsumura, R. and Akashi, M. (2014). The role of the endocrine system in feeding-induced tissue-specific circadian entrainment. Cell Rep 8(2): 393401.

8. Schibler, U., Gotic, I., Saini, C., Gos, P., Curie, T., Emmenegger, Y., Sinturel, F., Gosselin, P., Gerber, A., Fleury-Olela, F., Rando, G., Demarque, M. and Franken, P. (2015). Clock-talk: Interactions between central and peripheral circadian oscillators in mammals. Cold Spring Harb Symp Quant Biol 80: 223-232.

9. Stokkan, K. A., Yamazaki, S., Tei, H., Sakaki, Y. and Menaker, M. (2001). Entrainment of the circadian clock in the liver by feeding. Science 291(5503): 490-493.

10. Takahashi, J. S. (2017). Transcriptional architecture of the mammalian circadian clock. Nat Rev Genet 18(3): 164-179.

11. Yamaguchi, A., Matsumura, R., Matsuzaki, T., Nakamura, W., Node, K. and Akashi, M. (2017). A simple method using ex vivo culture of hair follicle tissue to investigate intrinsic circadian characteristics in humans. Sci Rep 7(1): 6824.

12. Yamazaki, S., Numano, R., Abe, M., Hida, A., Takahashi, R., Ueda, M., Block, G. D., Sakaki, Y., Menaker, M. and Tei, H. (2000). Resetting central and peripheral circadian oscillators in transgenic rats. Science 288(5466): 682-685.

13. Yoo, S. H., Yamazaki, S., Lowrey, P. L., Shimomura, K., Ko, C. H., Buhr, E. D., Siepka, S. M., Hong, H. K., Oh, W. J., Yoo, O. J., Menaker, M. and Takahashi, J. S. (2004). PERIOD2::LUCIFERASE real-time reporting of circadian dynamics reveals persistent circadian oscillations in mouse peripheral tissues. Proc Natl Acad Sci U S A 101(15): 5339-5346. 